\title{
Upregulation of miR-33b promotes endometriosis via inhibition of Wnt/ß-catenin signaling and ZEB1 expression
}

\author{
HAIYAN ZHANG ${ }^{1,2}$, GUANG LI $^{2}$, XIUGUI SHENG ${ }^{3}$ and SHIQIAN ZHANG ${ }^{1}$ \\ ${ }^{1}$ Department of Gynecology, Affiliated Qilu Hospital of Shandong University, Jinan, Shandong 250002; \\ ${ }^{2}$ Department of Gynecology Ward 1, Linyi City People's Hospital, Linyi, Shandong 276000; \\ ${ }^{3}$ Department of Gynecology, Chinese Academy of Medical Sciences Tumor Hospital, Beijing 100021, P.R. China
}

Received November 19, 2017; Accepted July 30, 2018

DOI: $10.3892 / \mathrm{mmr} .2019 .9870$

\begin{abstract}
The present study aimed to investigate the role and mechanisms of microRNA (miR)-33b in endometriosis (Ems). Reverse transcription-quantitative polymerase chain reaction (RT-qPCR), MTT assays, flow cytometry, caspase-3/9 activity assays and western blotting were performed in the present study. Initially, miR-33b expression in an Ems rat model was investigated by RT-qPCR and was demonstrated to be upregulated in Ems tissue samples of rats compared with the control group. In addition, miR-33b upregulation inhibited cell growth and enhanced apoptosis in an Ems model (primary cell cultures) compared with the control group. In addition, miR-33b up-regulation reduced Wnt/ $\beta$-catenin signaling pathway and suppressed zinc finger E-box-binding homeobox 1 (ZEB1) protein expression in the in vitro Ems model (primary cell cultures) compared with the control group. Furthermore, small interfering-ZEB1 ameliorated the effects of miR-33b downregulation on Ems cell growth in the in vitro Ems model. Additionally, a Wnt agonist reduced the effects of miR-33b upregulation on Ems cell growth in the in vitro Ems model. In conclusion, the present study demonstrated that upregulation of miR-33b may promote Ems through Wnt/ $\beta$-catenin by ZEB1 expression.
\end{abstract}

\section{Introduction}

Endometriosis (Ems) is a common benign gynecological disease in women of child-bearing age. It indicates that endometrial tissues (glands and mesenchyme) with growth function are coated with endometrium in the uterine cavity (1). Additionally, endometrial tissues may grow in other sites apart from the myometrium. Ems morbidity has notably

Correspondence to: Dr Shiqian Zhang, Department of Gynecology, Affiliated Qilu Hospital of Shandong University, 107 Culture West Road, Lixia, Jinan, Shandong 250002, P.R. China

E-mail: sxn183762@126.com

Key words: microRNA-33b, endometriosis, Wnt/ $\beta$-catenin, zinc finger E-box-binding homeobox 1 increased in recent years, with the major clinical symptoms of infertility, chronic pelvic pain and dysmenorrhea (1). Ems frequently occurs in the ovary, rectovaginal pouch and vesicouterine pouch (1). It is a benign disease, but certain of its biological behaviors resemble those of malignancies (2). It is extremely invasive, and may lead to extensive and severe adhesion. Additionally, Ems is regarded as a benign tumor, which severely affects patient quality of life (2).

microRNAs (miRNAs/miRs) are a class of endogenous RNAs with regulatory functions in eukaryotes (3). They are 22-23 nucleotides in length (4). miRNAs are extensively distributed in plants, animals and viruses, and negatively regulate gene expression at the post-transcriptional level through complementary pairing of mRNA (3). Eventually, they may lead to mRNA degradation or translational inhibition (5). As important regulatory molecules, miRNAs are involved in a series of vital life processes, such as virus defense, hematopoiesis, organogenesis, cell proliferation, apoptosis, fat metabolism and tumorigenesis (5).

The Wnt/ $\beta$-catenin signaling pathway is a key pathway regulating cell growth and proliferation (6). A previous study demonstrated that such a pathway serves a vital role in the genesis, metastasis and invasion of multiple tumors (6). In addition, it may be involved in the adhesion, invasion and angiogenesis of the ectopic endometrium (7). Wnt/ $\beta$-catenin signaling pathway serves a decisive role in endometrial gland formation and mesenchymal development (7).

Transcription factor zinc-finger E-box binding homeobox (ZEB)1 located in the short arm of human chromosome 10, is also referred to as TCF8 or $\delta E F 1$ (8). Recently, it was indicated that ZEB1 serves a crucial role in tumorigenesis (8) in different cancer types, including breast cancer, prostate cancer, lung cancer and endometrial cancer. Transcription factor ZEB-1 is one of the factors inducing Ems (9). A previous study on the role of ZEB1 in promoting tumor cell metastasis focused on its inhibitory effect on E-cadherin (10). Cadherins are a class of $\mathrm{Ca}^{2+}$-dependent transmembrane glycoproteins (10). E-cadherin marks epithelial cells and its deletion may be observed in Ems (10). Mesenchymal cell markers, including N-cadherin, are upregulated in Ems (11). Wang et al (12) demonstrated that miRNA-33b is able to mediate the cellular apoptosis of endometrial cells. The present study aimed to investigate the role and mechanism of miRNA-33b in Ems. 


\section{Materials and methods}

Animals and rat model. Female Sprague-Dawley rats (6-7 weeks old; $180-200 \mathrm{~g} ; \mathrm{n}=12$ ) were housed at $22-24^{\circ} \mathrm{C}$, $55-60 \%$ humidity with a 12 -h/12-h light/dark cycle, and were given a regular chow diet and water ad libitum. The present study was approved by the Animal Care and Use Committee of Affiliated Qilu Hospital of Shandong University (Jinan, China). All rats were randomly assigned to control $(n=6)$ and Ems $(n=6)$ groups. In the control group, rats were anesthetized with $35 \mathrm{mg} / \mathrm{kg}$ pentobarbital sodium without intervention. Rats were anesthetized with $35 \mathrm{mg} / \mathrm{kg}$ pentobarbital sodium, and a vertical incision in the abdomen was made. The uterus was removed and immediately washed with PBS, the endometrium was cut into $0.5 \times 0.5-\mathrm{cm}$ sections and uterine segments were sutured onto the peritoneum close to blood vessels. $0.5 \mu \mathrm{g} / \mathrm{kg} / \mathrm{day}$ of Estradiol benzoate (MedChem Express, Shanghai, China) was subcutaneously injected for 3 days following the surgery. Following treatment with Estradiol benzoate for 3 days, rats were sacrificed using decollation under $35 \mathrm{mg} / \mathrm{kg}$ pentobarbital sodium.

Cell culture and cell transfection. Endometrial stromal cells were separated from the isolated endometrial tissues, and tissue was finely minced. Cells were dispersed and incubated in Dulbecco's modified Eagle's medium (DMEM)/F-12 (Gibco; Thermo Fisher Scientific, Inc.), penicillin-streptomycin solution, and $2 \mathrm{mg} / \mathrm{ml}$ collagenase II for $1 \mathrm{~h}$ at $37^{\circ} \mathrm{C}$. Subsequently, endometrial stromal cells were separated using a $100 \mu \mathrm{m}$ filter. Stromal cells were pelleted by centrifugation at $200 \mathrm{x}$ g for 10 min at $4^{\circ} \mathrm{C}$ and incubated with DMEM/F-12 containing fetal bovine serum (10\%, v/v; Sigma-Aldrich; Merck KGaA, Darmstadt, Germany) at $37^{\circ} \mathrm{C}$ in a humidified atmosphere containing $5 \% \mathrm{CO}_{2}$. Then, transfection with $100 \mathrm{ng}$ of miR-33b (5'-GUGCAUUGCUGUUGCAUUGC-3' and 5'-AAUGCA ACAGCAAUGCACUU-3'), anti-miR-33b (forward, 5'-CCA AGGATCTCCAGGCTCGAA-3' and reverse, 5'-TTCGAG CCTGGAGATCCTTGG-3'), small interfering (si)-ZEB1 (sc-38643; Santa Cruz Biotechnology, Inc., Dallas, TX, USA) and negative mimics (forward, 5'-TTCTCCGAACGTGTCAC GT-3' and reverse, 5'-ACGTGACACGTTCGGAGAA-3') was performed using Lipofectamine ${ }^{\circledR} 2000$ (Invitrogen; Thermo Fisher Scientific, Inc., Waltham, MA, USA), according to the manufacturer's protocol. Following transfection for $4 \mathrm{~h}$, old medium was removed and new DMEM/F-12 was added into cell. Next, transfection of miR-33b or negative mimics was performed for $4 \mathrm{~h}$. For Wnt activation, $10 \mu \mathrm{M}$ of SKL2001 (MedChemExpress, Shanghai, China) was added to the cells for $72 \mathrm{~h}$.

RNA extraction and reverse transcription-quantitative polymerase chain reaction ( $R T-q P C R)$. Total RNA was extracted from frozen tissues or cells using TRIzol ${ }^{\circledR}$ reagent (Life Technologies; Thermo Fisher Scientific, Inc.). Total RNA $(1 \mu \mathrm{g})$ was transcribed to first-strand cDNA using an RT kit (Toyobo Life Science, Osaka, Japan). The RT-qPCR protocol was performed using SYBR-Green PCR master mix (Bio-Rad Laboratories, Hercules, CA, USA) and an Applied Biosystems 7300 Real-Time PCR System (Thermo Fisher Scientific, Inc.). The primer sequences were: miR-33b forward, 5'-ATT
CTTTCGAACTGTCTTGG-3' and reverse, 5'-TCACCCTCG GCTGTCCTGACA-3'; U6 forward, 5'-AGTACCAGTCTG TTGCTGG-3' and reverse, 5'-TAATAGACCCGGATGTCT GGT-3'. The qPCR cycle was set to an initial $95^{\circ} \mathrm{C}$ for $10 \mathrm{~min}$, followed by 40 cycles of $95^{\circ} \mathrm{C}$ for $25 \mathrm{sec}, 60^{\circ} \mathrm{C}$ for $30 \mathrm{sec}$, and $72^{\circ} \mathrm{C}$ for $30 \mathrm{sec}$. Gene expression levels were measured using the $2^{-\Delta \Delta \mathrm{Cq}}$ method (13).

Gene microarray hybridization. Isolated RNA was reverse transcribed into cDNA and hybridized to Affymetrix HG-U133 Plus 2.0 GeneChip arrays 1 (Affymetrix, Santa Clara, CA, USA). Data were analyzed through TargetScan version 7.1 (http://www.targetscan.org) and QIAGEN's Ingenuity Pathway Analysis (IPA, QIAGEN, Redwood City, USA) (14).

Hematoxylin and eosin $(H \& E)$ staining. Endometriosis tissue was collected and fixed with $4 \%$ paraformaldehyde for $24 \mathrm{~h}$. The colonic sections of $4 \mu \mathrm{m}$ were cut from formalin-fixed, paraffin-embedded tissue blocks. Tissue samples were stained with HE assay for 15 min and evaluated under the light microscope (Olympus BX51).

Cell viability and apoptosis. MTT (5 mg/ml; Sigma Aldrich; Merck KGaA) was added to the cells for incubation at $37^{\circ} \mathrm{C}$ for $4 \mathrm{~h}$. Subsequently, the old medium was removed and dimethyl sulfoxide was added to the cells for $20 \mathrm{~min}$ at $37^{\circ} \mathrm{C}$. The absorbance was measured by spectrophotometry with a microplate reader (model 680; Bio-Rad Laboratories, Inc.) at $490 \mathrm{~nm}$. Endometrial stromal cells were washed with PBS and resuspended in $100 \mu \mathrm{l} 1 \mathrm{X}$ binding buffer (BestBio, Shanghai, China) and incubated with Annexin V-fluorescein isothiocyanate (FITC; BestBio) and propidium iodide (PI; BestBio) for $15 \mathrm{~min}$ at room temperature in the dark. The apoptosis rate was analyzed using a flow cytometer (C6; Beckman Coulter Inc., Brea, CA, USA) analyzed using Image Lab version 3.0 (Bio-Rad Laboratories, Inc.).

Lactate dehydrogenase (LDH) activity. LDH activity levels were measured using LDH activity kits (C0016; Beyotime Institute of Biotechnology, Haimen, China). The absorbance was measured by spectrophotometry with a microplate reader (model 680; Bio-Rad Laboratories, Inc.) at $450 \mathrm{~nm}$.

Caspase 3/9 activity assay. Endometrial stromal cells were lysed with lysis buffer (radioimmunoprecipitation assay buffer) containing a protease inhibitor cocktail (phenylmethanesulfonyl fluoride) and EDTA at $4^{\circ} \mathrm{C}$ for $30 \mathrm{~min}$. Cells were centrifuged at $12,000 \mathrm{x} \mathrm{g}$ for $10 \mathrm{~min}$ at $4^{\circ} \mathrm{C}$. Subsequently, the concentration of total protein was determined using a bicinchoninic acid (BCA) assay, and $10 \mu \mathrm{g} /$ lane total protein was used to analyze caspase $3 / 9$ activity levels using caspase 3/9 activity kits (C1115 and C1158, Beyotime Institute of Biotechnology). The absorbance was measured by spectrophotometry with a microplate reader (model 680; Bio-Rad Laboratories) at $405 \mathrm{~nm}$.

Western blot analysis. Endometrial stromal cells were lysed with lysis buffer (radioimmunoprecipitation assay buffer) containing protease inhibitor cocktail (phenylmethanesulfonyl fluoride) and EDTA at $4^{\circ} \mathrm{C}$ for $30 \mathrm{~min}$. Cells were centrifuged 


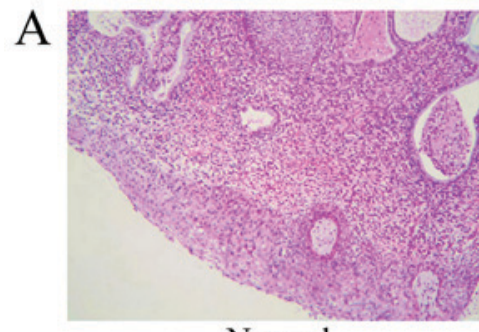

Normal

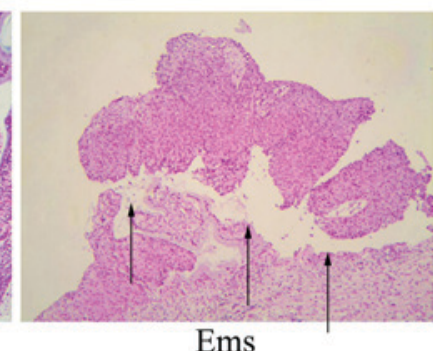

Ems

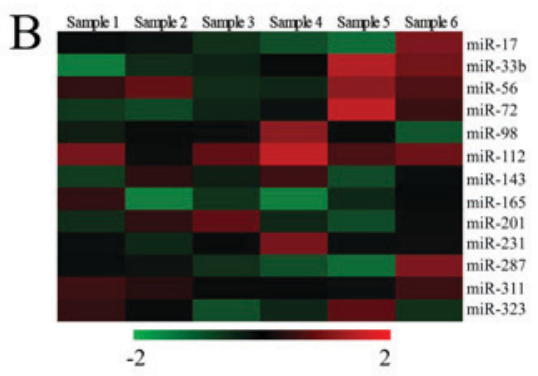

$-2$

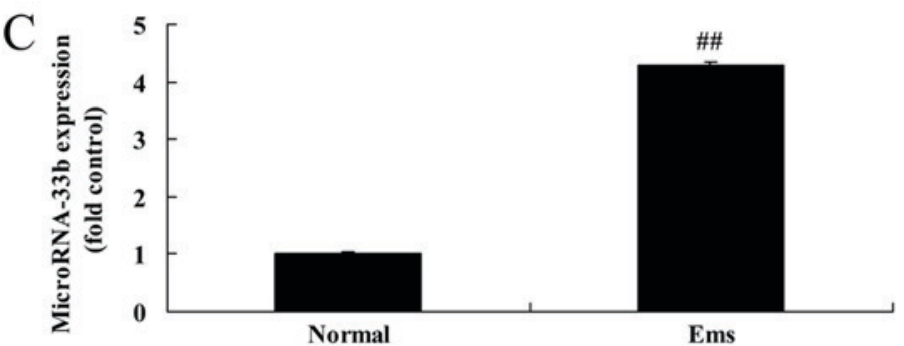

Figure 1. Expression of miR-33b in Ems. (A) Hematoxylin and eosin staining for Ems (magnification, x100). (B) Heat map for miR expression and (C) quantification of miR-33b expression as measured by RT-qPCR. ${ }^{\#}$ P $<0.05$ vs. normal. Ems, endometriosis; miR, microRNA.

at $12,000 \mathrm{x} \mathrm{g}$ for $10 \mathrm{~min}$ at $4^{\circ} \mathrm{C}$. Subsequently, the concentration of total protein was determined using the BCA assay, and $40 \mu \mathrm{g} /$ lane total protein was subjected to $10-12 \%$ SDS-PAGE and subsequently transferred onto a polyvinylidene difluoride membrane (Bio-Rad Laboratories, Inc.). The membrane was blocked with $5 \%$ non-fat milk in TBS containing $0.1 \%$ Tween-20 for $1 \mathrm{~h}$ at $37^{\circ} \mathrm{C}$, and subsequently incubated with antibodies against Wnt (sc-376029; 1:1,000), $\beta$-catenin (sc-65480; 1:1,000), ZEB1 (sc-515797; 1:1,000), apoptosis regulator BAX (Bax; sc-20067; 1:1;000) and GAPDH (sc-51631; 1:5,000; all from Santa Cruz Biotechnology, Inc., Dallas, TX, USA) overnight at $4^{\circ} \mathrm{C}$. Following this, membranes were incubated with anti-rabbit immunoglobulin G secondary antibody (sc-2004; 1:5,000; Santa Cruz Biotechnology, Inc.) at $37^{\circ} \mathrm{C}$ for $1 \mathrm{~h}$ and were developed using an enhanced chemiluminescence kit (GE Healthcare Bio-Sciences, Pittsburgh, PA, USA). Protein levels were quantified using Bio-Rad Laboratories, Inc. Quantity One software (version 3.0).

Statistical analysis. All data are expressed as the mean \pm standard error using SPSS version 17.0 (SPSS, Inc., Chicago, IL, USA). Statistical differences were measured using one way analysis of variance with Bonferroni's correction for multiple comparisons. $\mathrm{P}<0.05$ was considered to indicate a statistically significant difference.

\section{Results}

miR-33b expression in Ems. Firstly, H\&E staining suggested that Ems was successfully induced in the Ems group compared with the control group (Fig. 1A). As demonstrated in Fig. 1B and $\mathrm{C}, \mathrm{miR}-33 \mathrm{~b}$ expression was upregulated by $4.29 \pm 0.05$ fold in the Ems rat model compared with the control group.

Effect of miR-33b on cell growth in Ems. Notably, miR-33b expression was investigated using miR-33b mimics or anti miR-33b mimics in vitro. Fig. $2 \mathrm{~A}$ and $\mathrm{B}$ indicate that miR-33b expression was upregulated by $5.26 \pm 0.04$ fold or downregulated by $0.26 \pm 0.03$ fold in the in vitro Ems model compared with the control group. Overexpression of miR-33b suppressed cell viability by $0.82 \pm 0.06$ fold $(48 \mathrm{~h}$ ) or $0.51 \pm 0.06$ fold $(72 \mathrm{~h}$ ), and enhanced the lactate dehydrogenase (LDH) activity of Ems by $4.70 \pm 0.29$ fold; additionally, downregulation of miR-33b promoted cell viability by $1.23 \pm 0.06$ fold (48 h) or $1.28 \pm 0.03$ fold ( $72 \mathrm{~h}$ ), and decreased the LDH activity of Ems by $0.37 \pm 0.03$ fold (Fig. 2C-F).

Effect of miR-33b on apoptosis in Ems. Subsequently, it was demonstrated that overexpression of miR-33b increased the apoptosis rate by $2.05 \pm 0.15$-fold and promoted caspase- 3 and 9 activity by $3.97 \pm 0.17$ and $3.70 \pm 0.24$-fold, respectively, in the Ems model compared with the control group (Fig. 3A-D). However, downregulation of miR-33b decreased the apoptosis rate by $0.54 \pm 0.04$-fold (Fig. $3 \mathrm{E}$ and F) and the caspase- 3 and 9 activity by $0.39 \pm 0.03$ and $0.31 \pm 0.05$-fold, respectively, in the Ems model (Fig. 3G and H).

Effect of miR-33b on Wnt/ $\beta$-catenin in Ems by ZEB1 expression. To analyze the mechanism of miR-33b in Ems, the Wnt/ $\beta$-catenin signaling pathway was investigated. It was demonstrated that miR-33b was able to target the 3'UTR of ZEB1 (Fig. 4A). Subsequently, overexpression of miR-33b suppressed ZEB1 protein expression by $0.34 \pm 0.04$ fold, reduced $\mathrm{Wnt}$ and $\beta$-catenin protein expression by $0.44 \pm 0.06$ and $0.32 \pm 0.12$ fold, respectively, and induced Bax protein expression by $2.56 \pm 0.13$ fold in the in vitro Ems model (Fig. 4B-F). Furthermore, downregulation of miR-33b induced ZEB1 protein expression by $1.94 \pm 0.17$ fold, reduced Wnt/ $\beta$-catenin protein expression by $2.57 \pm 0.07$ and $2.72 \pm 0.14$ fold, respectively, and suppressed Bax protein expression by $0.43 \pm 0.02$ fold in the in vitro Ems model compared with the control group (Fig. 4G-K).

$Z E B 1 / W n t / \beta$-catenin influences the effect of miR-33b in Ems. To examine the role of ZEB1/Wnt/ $\beta$-catenin in the effect of miR-33b on Ems, si-ZEB1 or Wnt agonist were co-transfected with 

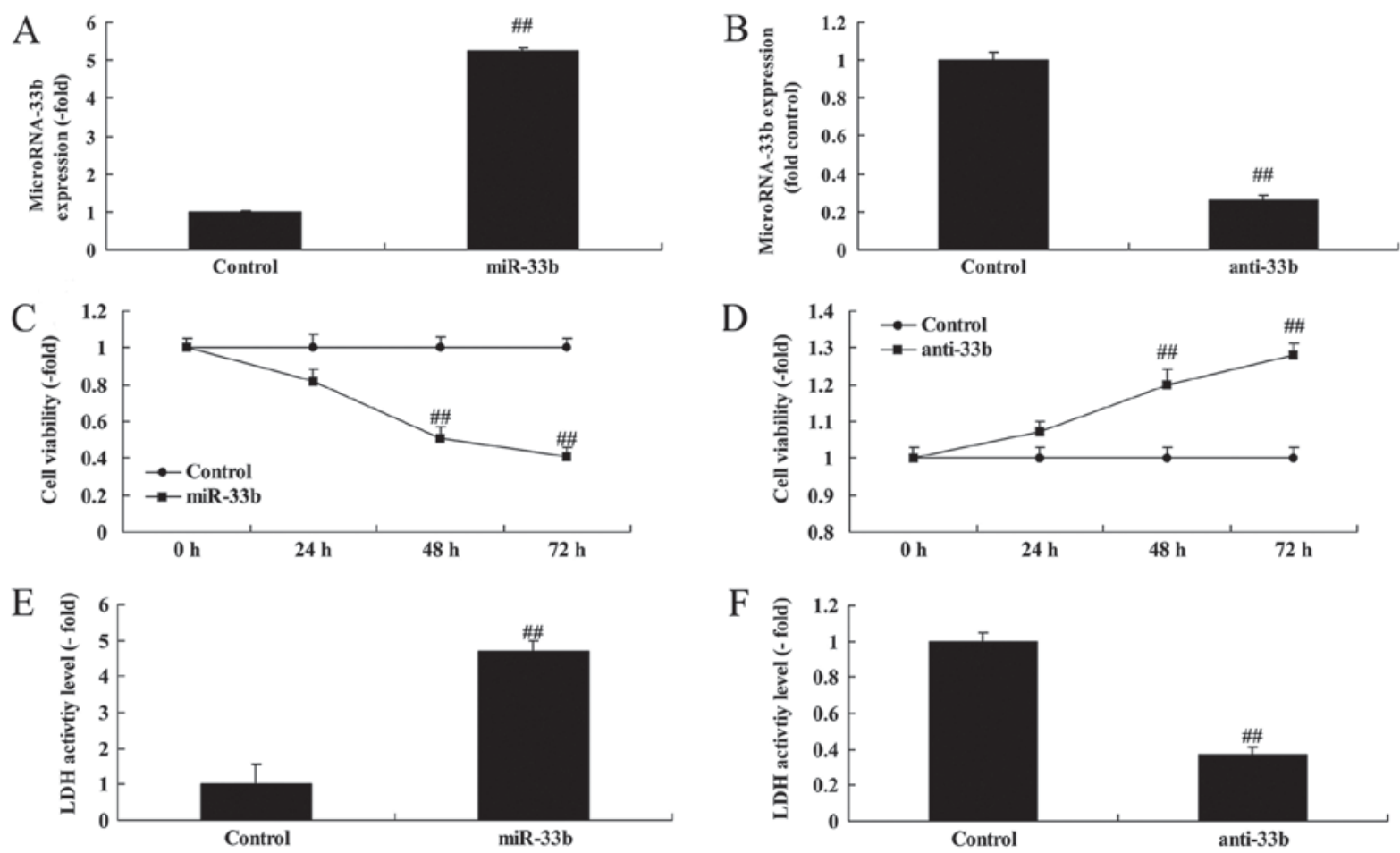

Figure 2. Effect of miR-33b on cell growth in endometriosis. Quantification of miR-33b expression following treatment with its (A) mimic and (B) anti-mimic as measured by RT-qPCR. Effect of (C) miR-33b mimic and (D) anti-mimic on cell viability. Effect of (E) miR-33b mimic and (F) anti-mimic on LDH activity levels. ${ }^{\#} \mathrm{P}<0.05$ vs. respective control. miR, microRNA. LDH, lactate dehydrogenase.
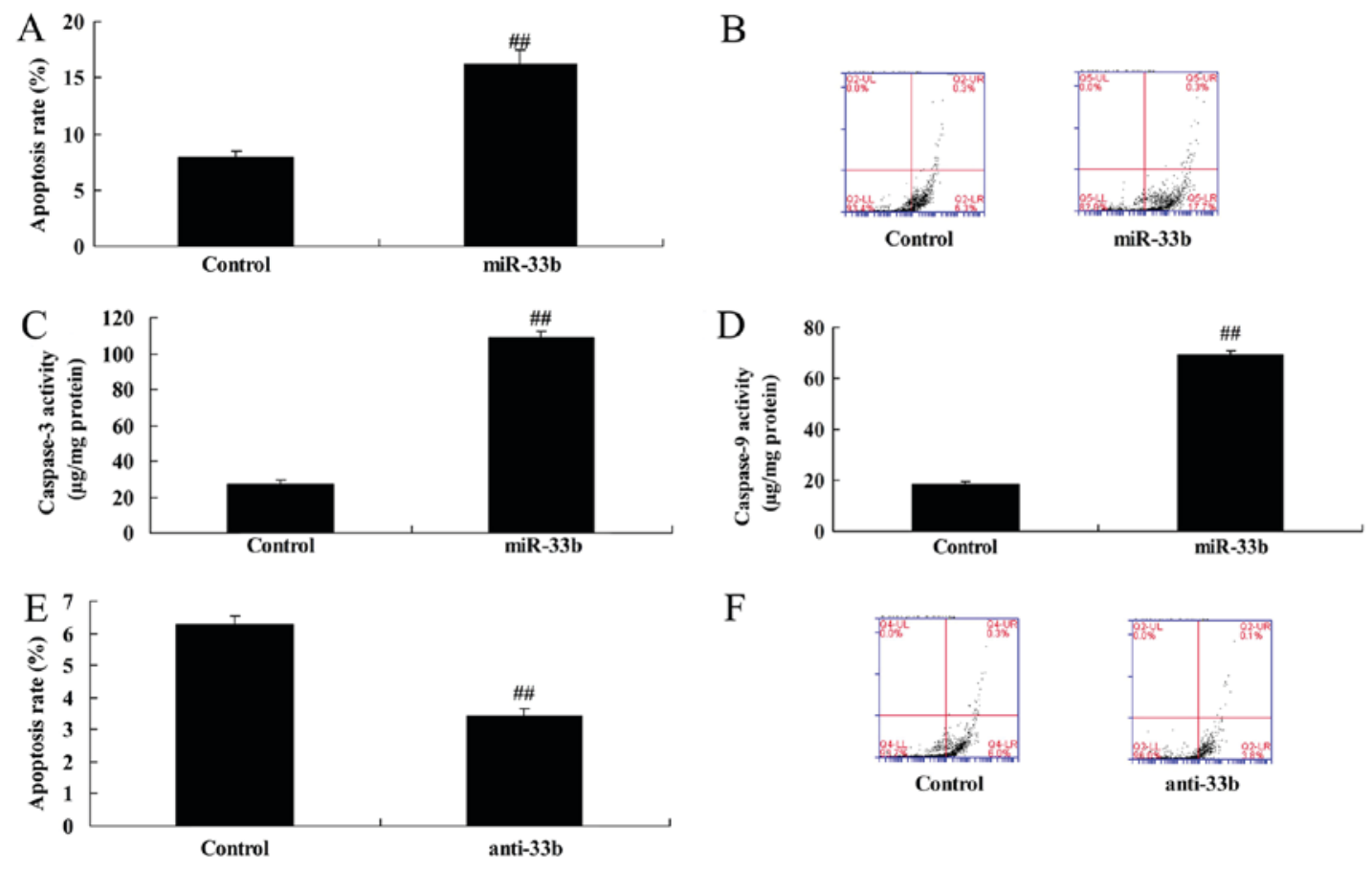

F
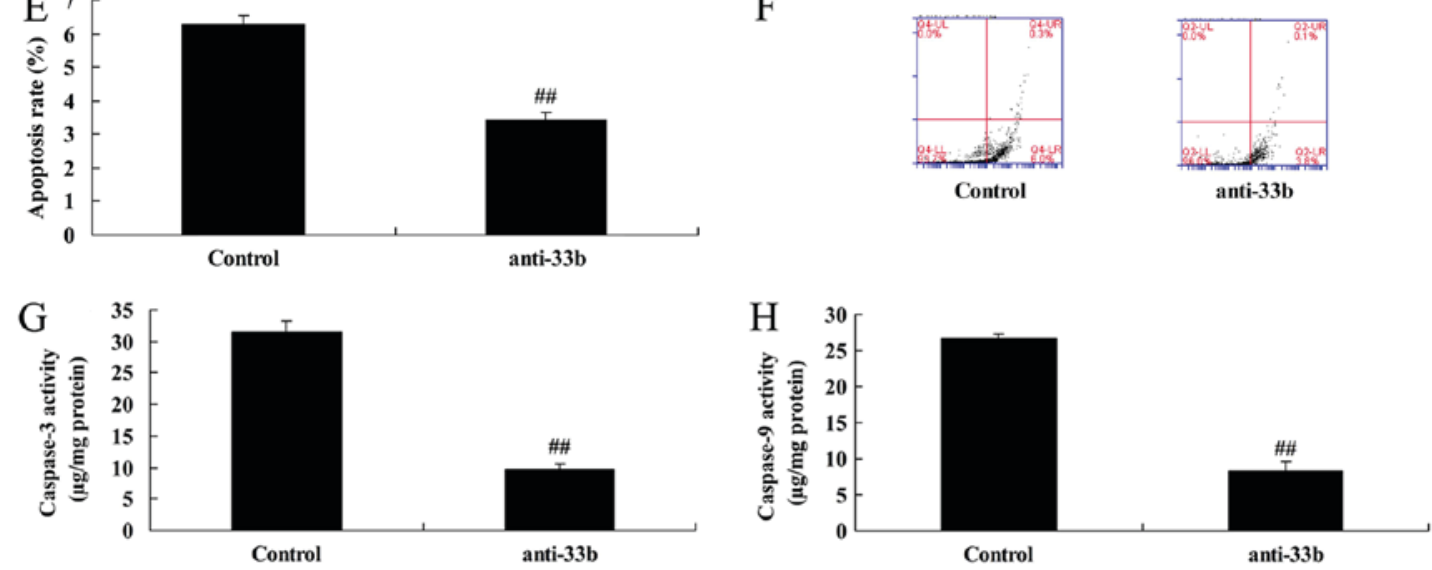

Figure 3. Effect of miR-33b on apoptosis in Ems. Effects of overexpression of miR-33b on (A and B) apoptotic rate, (C and D) caspase-3 and caspase-9 activity respectively; and of downregulation of miR-33b on ( $\mathrm{E}$ and $\mathrm{F})$ apoptotic rate, $(\mathrm{G}$ and $\mathrm{H})$ caspase-3 and caspase- 9 activity, respectively. ${ }^{\sharp \#} \mathrm{P}<0.05$ vs. control. Ems, endometriosis; miR, microRNA. 
A ZEB1: ...AAUGCAU...
miR-33b: ...UUACGUG...
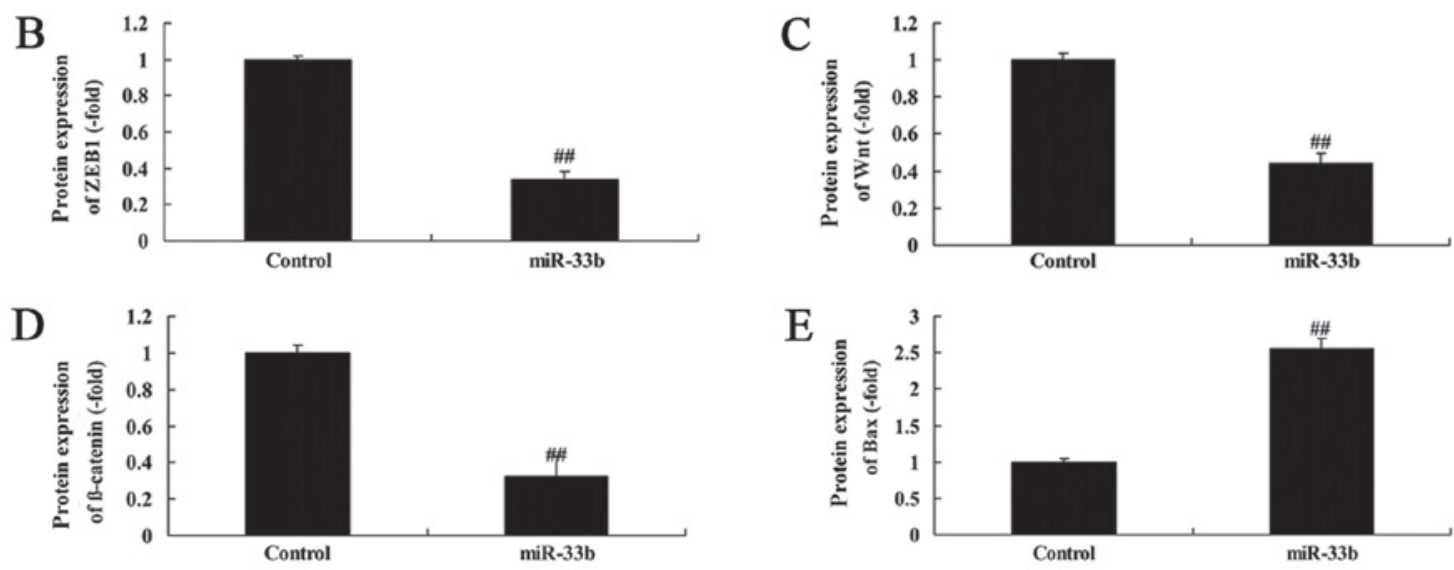

F
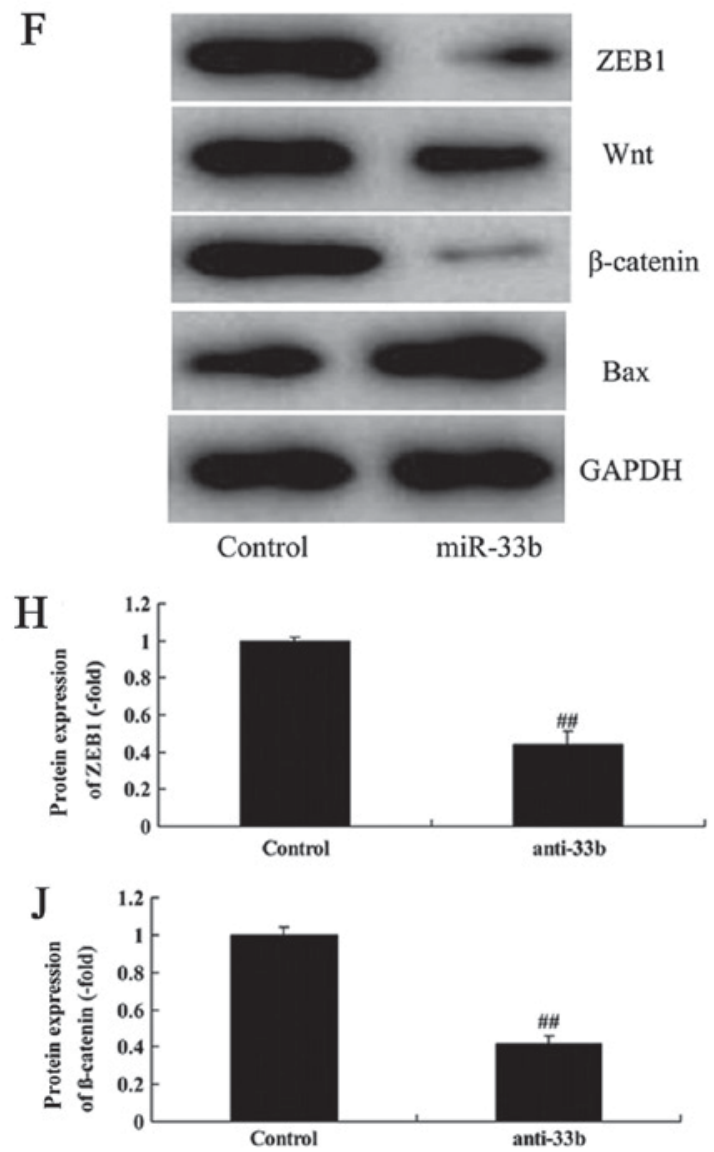

G

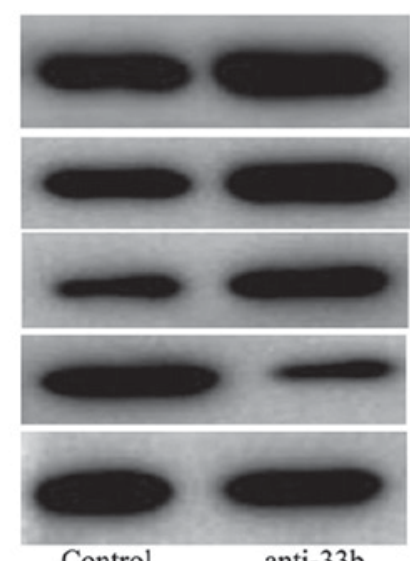

ZEB 1

Wnt

$\beta$-catenin

Bax

GAPDH
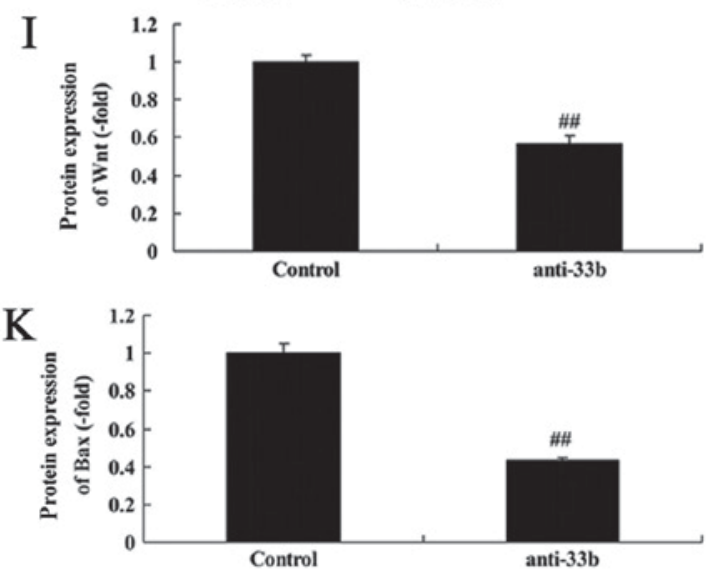

Figure 4. Effect of miR-33b on Wnt/ $\beta$-catenin in Ems by ZEB1 expression. (A) miR-33b targets the 3'untranslated region of ZEB1. Quantification of (B) ZEB1, (C) Wnt, (D) $\beta$-catenin and (E) Bax protein expression levels, following overexpression of miR-33b. (F) Representative western blotting image. (G) Western blot analysis and quantification of (H) ZEB1, (I) Wnt, (J) $\beta$-catenin and (K) Bax protein expression levels, following downregulation of miR-33b. ${ }^{\# \#} \mathrm{P}<0.05$ vs. control. Bax, apoptosis regulator BAX; Ems, endometriosis; miR, microRNA; ZEB1, zinc-finger E-box binding homeobox 1.

miR-33b mimics in Ems. As illustrated in Fig. 5A-E, compared with the miR-33b downregulation group, si-ZEB1 suppressed ZEB1 by $0.52 \pm 0.08$ fold, decreased Wnt and $\beta$-catenin protein expression by $0.57 \pm 0.04$ and $0.71 \pm 0.05$ fold, respectively, and induced Bax protein expression by $2.36 \pm 0.07$ fold in the Ems model. Furthermore, compared with the miR-33b overexpression group, the Wnt agonist induced Wnt and $\beta$-catenin protein expression by $2.01 \pm 0.06$ and $1.61 \pm 0.05$ fold, respectively, and suppressed Bax protein expression by $0.61 \pm 0.07$-fold in the in vitro Ems model compared with miR-33b (Fig. 5F-I).

ZEB1 inhibition reduces the effect of anti-miR-33b on cell viability in Ems. To gain insight into the function of miR-33b on Ems, the effect of the ZEB1 inhibitor on the function of miR-33b in Ems was analyzed. Compared with the miR-33b downregulation group, ZEB1 inhibition reduced cell viability 

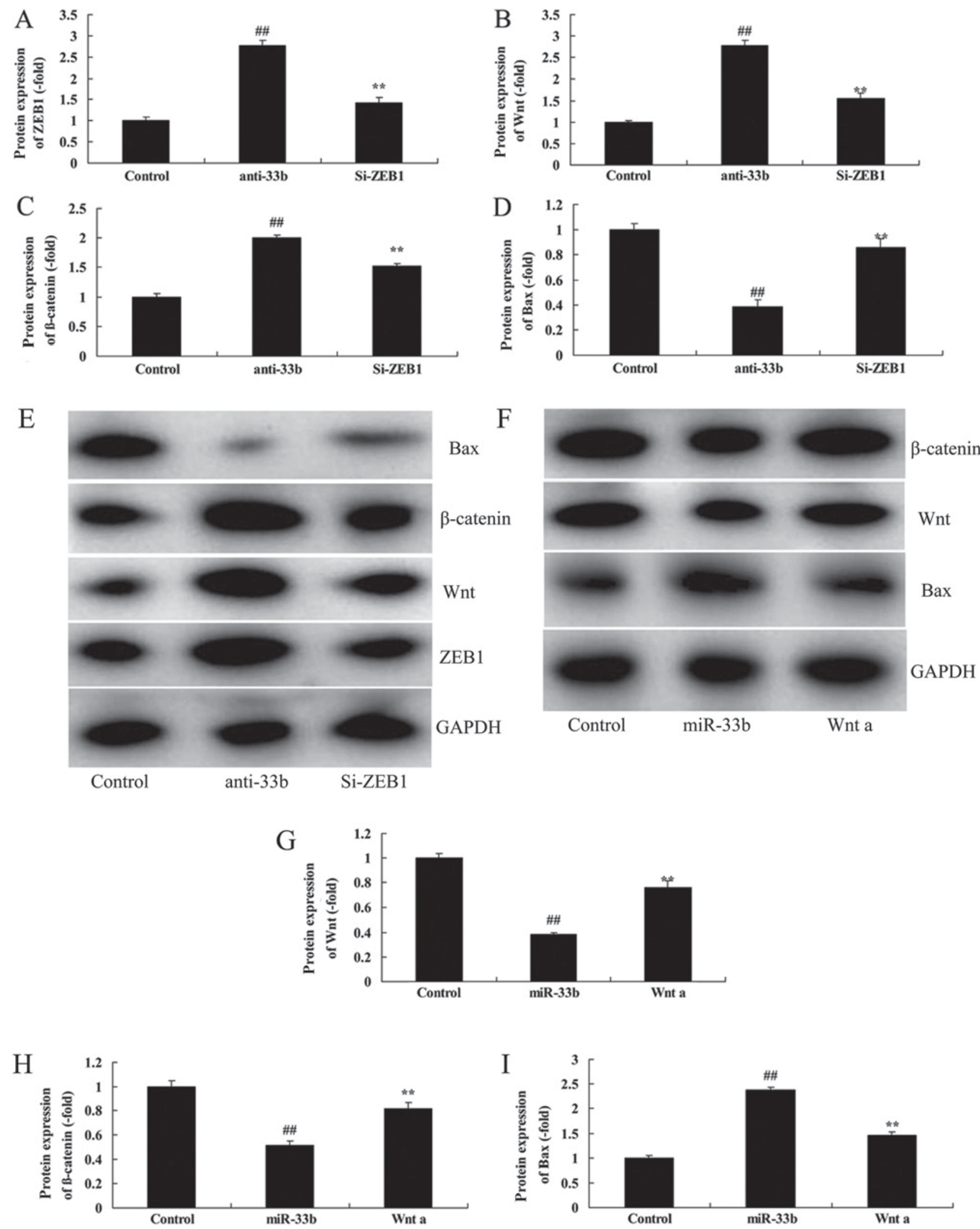

Figure 5. ZEB1/Wnt/ $\beta$-catenin mediates the effect of miR-33b in Ems. Quantification of (A) Bax, (B) $\beta$-catenin, (C) Wnt and (D) ZEB1 protein expression levels, following downregulation of miR-33b. ${ }^{\# \#} \mathrm{P}<0.05$ vs. control; ${ }^{* *} \mathrm{P}<0.05$ vs. anti-33b. (E) Representative western blotting image. (F) Western blot analysis and quantification of $(\mathrm{G}) \mathrm{Wnt},(\mathrm{H}) \beta$-catenin, (I) and Bax protein expression levels, following overexpression of miR-33b and treatment with a Wnt agonist. ${ }^{\# \# /} \mathrm{P}<0.05$ vs. control; ${ }^{* *} \mathrm{P}<0.05$ vs. miR-33b. Bax, apoptosis regulator BAX; Ems, endometriosis; miR, microRNA; si, small interfering; ZEB, zinc-finger E-box binding homeobox.

by $0.81 \pm 0.05(48 \mathrm{~h})$ or $0.71 \pm 0.08$-fold $(72 \mathrm{~h})$, increased LDH activity by $2.12 \pm 0.04$ fold, increased caspase- 3 and 9 activity by $2.72 \pm 0.14$ and $2.16 \pm 0.12$-fold, respectively, and apoptosis rate by $2.26 \pm 0.07$ fold in the in vitro Ems model (Fig. 6). 

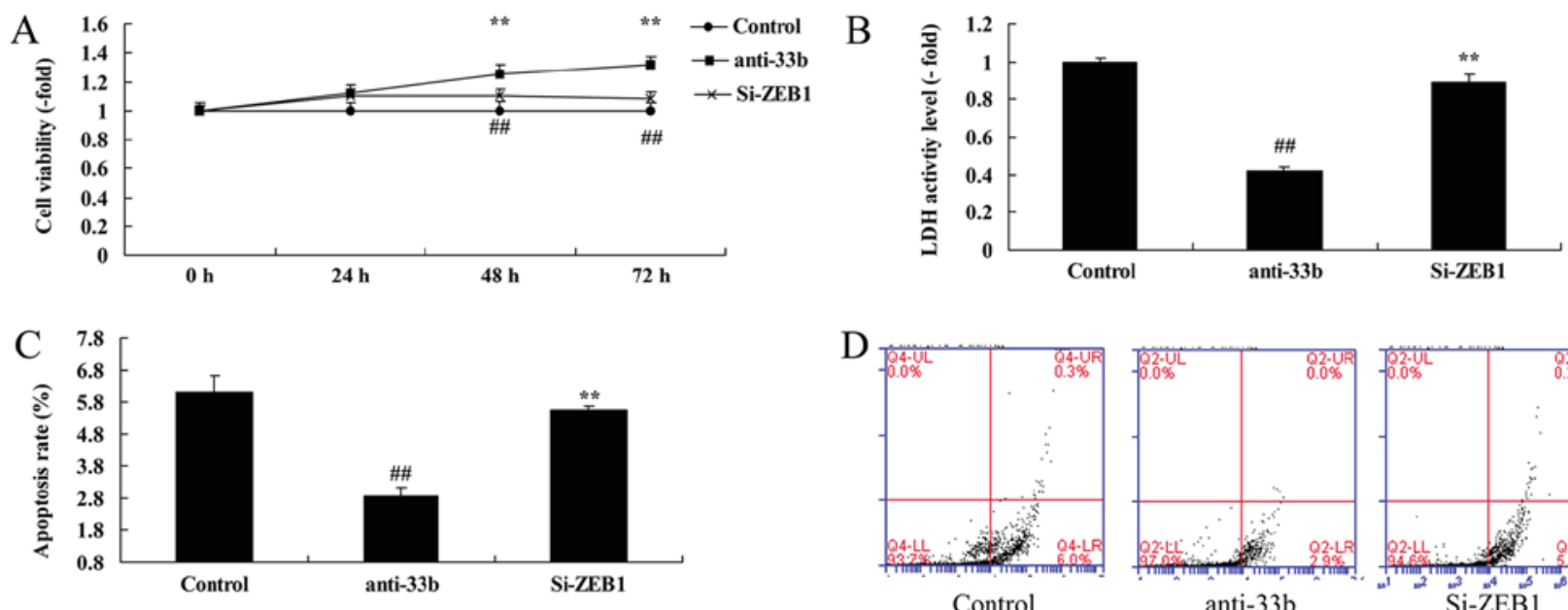

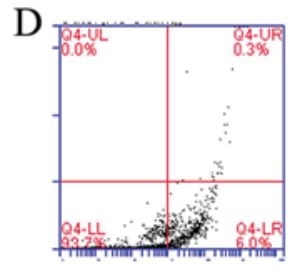

Control

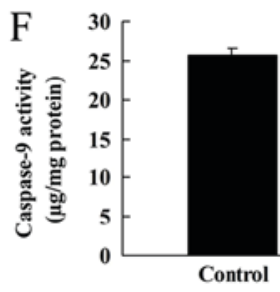

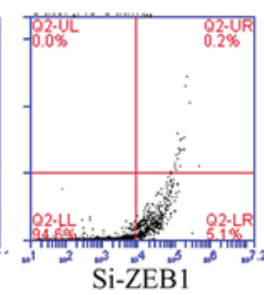

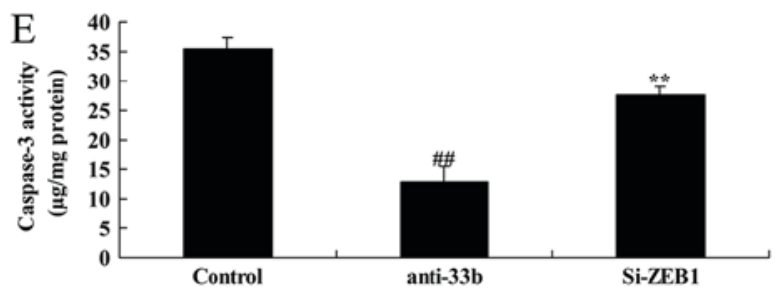

Figure 6. Inhibition of ZEB1 reduces the effect of anti-miR-33b on cell growth in Ems. Effects of downregulation of miR-33b and of ZEB1 on (A) cell viability, (B) LDH activity, (C) apoptosis rate as assessed by (D) flow cytometry, and on (E) caspase-3 and (F) caspase-9 activity. ${ }^{\# \#} \mathrm{P}<0.05$ vs. control;

${ }^{* *} \mathrm{P}<0.05$ vs. anti-33b. Ems, endometriosis; miR, microRNA; si, small interfering; ZEB, zinc-finger E-box binding homeobox; LDH, lactate dehydrogenase.
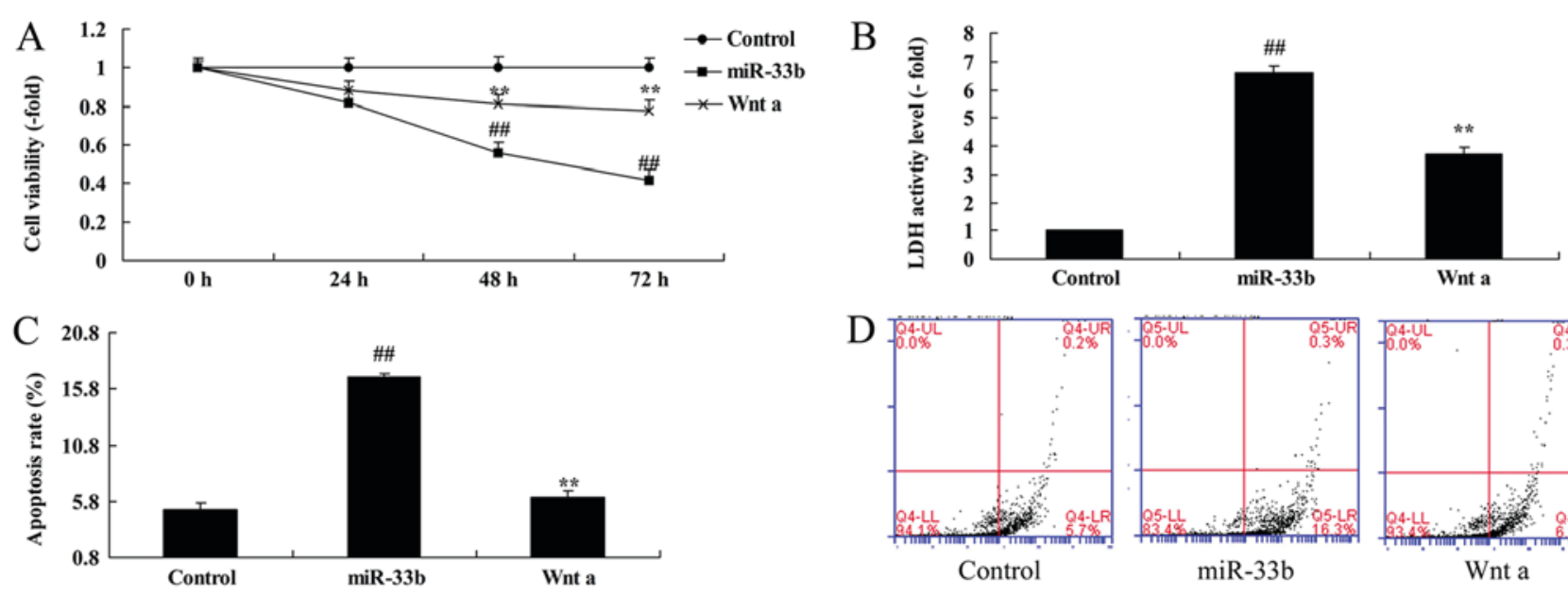

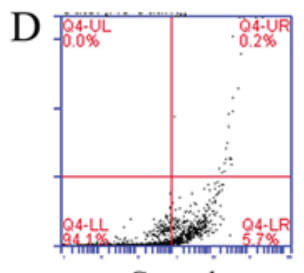

Control

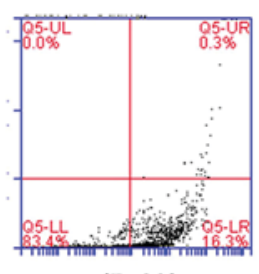

miR-33b
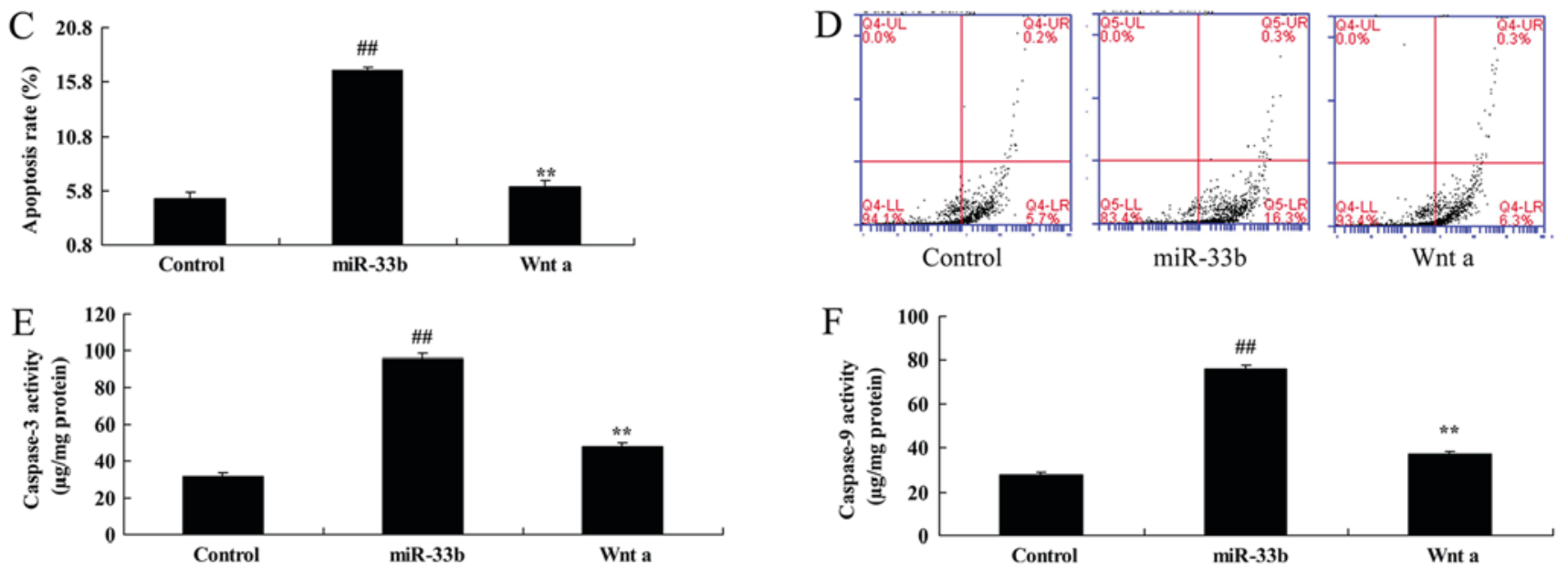

Figure 7. Activation of Wnt inhibits the effect of miR-33b on cell viability of Ems. Effects of overexpression of miR-33b and of treatment with a Wnt agonist on (A) cell viability, (B) LDH activity, (C) apoptosis rate as assessed by (D) flow cytometry, and on (E) caspase-3 and (F) caspase-9 activity. ${ }^{\# \#} \mathrm{P}<0.05$ vs. control; ${ }^{* *} \mathrm{P}<0.05$ vs. anti-33b. Ems, endometriosis; miR, microRNA; LDH, lactate dehydrogenase.

Wnt activation inhibits the effect of miR-33b on cell viability in Ems. Furthermore, miR-33b mimics were co-transfected with Wnt agonist in the in vitro Ems model. Compared with the miR-33b overexpression group, Wnt activation inhibited cell growth by $1.46 \pm 0.06$ (48 h) or $1.86 \pm 0.05$ fold $(72 \mathrm{~h}), \mathrm{LDH}$ activity by $0.36 \pm 0.13$ fold, caspase- 3 and 9 activity by $0.39 \pm 0.03$ and $0.48 \pm 0.12$ fold, respectively, and apoptosis rate by $0.61 \pm 0.07$ fold in the in vitro Ems model following miR-33b (Fig. 7). 


\section{Discussion}

Ems is a common gynecological disease, which has similar features to malignancies, including cell growth, invasion, distant metastasis and recurrence, affecting the quality of life of patients (15). Ems is a benign disease with malignant behavior. Its morbidity has exhibited an increasing trend (15). However, its pathogenesis remains unclear (15). miRNAs are endogenous RNAs with regulatory functions discovered in eukaryotes and are able to suppress gene expression at the transcriptional level (5). As important regulatory molecules, miRNAs are involved in a series of vital life processes, including antiviral defense, hematopoiesis, organogenesis, cell proliferation, apoptosis, fat metabolism and tumorigenesis (5). In the present study, it was demonstrated that miR-33b expression was upregulated in an Ems rat model. Wang and Ren (12) demonstrated that miRNA-33b is able to mediate the apoptosis of endometrial stromal cells. In the present study, miR-33b expression was examined in a rat model, and miR-33b expression in vivo may be analyzed in further studies.

A previous study has indicated that the $\mathrm{Wnt} / \beta$-catenin signaling pathway may induce epithelial-mesenchymal transition (EMT) in epithelial cells (6). Research on the interaction between $\beta$-catenin and estrogen is available. The Wnt/ $\beta$-catenin signaling pathway is highly conserved (16). Additionally, a previous study has verified that the Wnt/ $\beta$-catenin signaling pathway serves a crucial role in ectopic endometrial cell adhesion, invasion and angiogenesis (17). The intracellular accumulation of $\beta$-catenin accounts for its principal mechanism of action (17). It activates its downstream target genes, including vascular epithelial growth factor and matrix metalloproteinases (MMPs), leading to abnormal cell proliferation, differentiation and maturation (18). Of these genes, MMP-9 is able to degrade extracellular matrix components, including type IV and type V collagen and gelatin (19). In addition, the Wnt/ $\beta$-catenin signaling pathway may promote vascular endothelial cell growth, thus leading to angiogenesis (6). Furthermore, the $\mathrm{Wnt} / \beta$-catenin signaling pathway is able to strengthen intercellular adhesion through mutual activation with integrin and, as a result, it serves a key role in ectopic adhesion, planting and the growth of endometrial cells (6). The present study demonstrated that overexpression of miR-33b may suppress ZEB1 protein expression and may reduce $\mathrm{Wnt} / \beta$-catenin protein expression in Ems in vitro. Wang et al (20) demonstrated that miRNA-33b inhibited lung adenocarcinoma cell growth and invasion by suppressing the Wnt/ $\beta$-catenin/ZEB1 signaling pathway. These results are consistent with the present ones, demonstrating that miRNA-33b may regulate the ZEB1/Wnt/ $\beta$-catenin signaling pathway to induce cell death in Ems.

High ZEB1 expression has been demonstrated in numerous malignancies, including lung, colorectal, prostate and ovarian cancer (21). ZEB1 is able to promote the malignant phenotype of Ems primarily by regulating EMT (11). Similarly, high ZEB1 expression may be detected in breast cancer, and may regulate uterine cell adhesion and polarity alterations (21). Furthermore, it may promote the abnormal proliferation of uterine stem cells (22). A previous study demonstrated thatZEB1 may regulate estrogen receptor- $\alpha$ silencing (22). A previous study indicated that ZEB1 may also be involved in epigenetic regulation during Ems (21). In the present study, it was demonstrated that ZEB1 inhibition decreased the effect of anti-miR-33b on cell growth in Ems. Wang et al (20) demonstrated that miRNA-33b inhibited lung adenocarcinoma cell growth and invasion by suppressing Wnt/ $\beta$-catenin/ZEB1 signaling. Wang et al (12) reported that the miR-33b/HMGA2/Twist1/ZEB1 axis serves a critical role in regulating melanoma dissemination. The results of the present study suggested that miRNA-33b/ZEB1/Wnt/ $\beta$-catenin inhibited cell growth in an Ems rat model. However, the downstream molecular pathway of miRNA-33b/ZEB1/Wnt/ $\beta$-catenin requires further study.

In conclusion, the present results demonstrated that upregulation of miRNA-33b may promote Ems via Wnt/ $\beta$-catenin by ZEB1 expression. Thus, restoration of miRNA-33b expression may be used as a novel strategy for the treatment of Ems, although this requires further investigation.

\section{Acknowledgements}

Not applicable.

Funding

No funding was received.

\section{Availability of data and materials}

The analyzed data sets generated during the study are available from the corresponding author on reasonable request.

\section{Authors' contributions}

SZ designed the experiment; HZ, GL and XS performed the experiment; SZ and HZ analyzed the data; SZ wrote the manuscript.

\section{Ethics approval and consent to participate}

The present study was approved by the Animal Care and Use Committee of Affiliated Qilu Hospital of Shandong University.

\section{Patient consent for publication}

Not applicable.

\section{Competing interests}

The authors declare that they have no competing interests.

\section{References}

1. Kang JL, Wang XX, Nie ML and Huang XH: Efficacy of gonadotropin-releasing hormone agonist and an extended-interval dosing regimen in the treatment of patients with adenomyosis and endometriosis. Gynecol Obstet Invest 69: 73-77, 2010.

2. Soto E, Luu TH, Liu X, Magrina JF, Wasson MN, Einarsson JI, Cohen SL and Falcone T: Laparoscopy vs. Robotic Surgery for Endometriosis (LAROSE): A multicenter, randomized, controlled trial. Fertil Steril 107: 996-1002 e1003, 2017.

3. Nematian SE, Mamillapalli R, Kadakia TS, Majidi Zolbin M, Moustafa S and Taylor HS: Systemic inflammation induced by microRNAs: Endometriosis derived alterations in circulating microRNA 125b-5p and let7b-5p regulate macrophage cytokine production. J Clin Endocrinol Metab 103: 64-74, 2018. 
4. Shen L, Yang S, Huang W, Xu W, Wang Q, Song Y and Liu Y MicroRNA23a and microRNA23b deregulation derepresses SF-1 and upregulates estrogen signaling in ovarian endometriosis. J Clin Endocrinol Metab 98: 1575-1582, 2013.

5. Wright KR, Mitchell B and Santanam N: Redox regulation of microRNAs in endometriosis-associated pain. Redox Biol 12: 956-966, 2017.

6. Zhang L, Xiong W, Xiong Y, Liu H, Li N, Du Y and Liu Y: Intracellular wnt/beta-catenin signaling underlying 17beta-estradiol-induced matrix metalloproteinase 9 expression in human endometriosis. Biol Reprod 94: 70, 2016.

7. Pazhohan A, Amidi F, Akbari-Asbagh F, Seyedrezazadeh E, Farzadi L, Khodarahmin M, Mehdinejadiani S and Sobhani A: The Wnt/ $\beta$-catenin signaling in endometriosis, the expression of total and active forms of $\beta$-catenin, total and inactive forms of glycogen synthase kinase- $3 \beta$, WNT7a and DICKKOPF-1. Eur J Obstet Gynecol Reprod Biol 220: 1-5, 2017.

8. Zhang P, Sun Y and Ma L: ZEB1: At the crossroads of epithelial-mesenchymal transition, metastasis and therapy resistance. Cell Cycle 14: 481-487, 2015.

9. Schmalhofer O, Brabletz S and Brabletz T: E-cadherin, beta-catenin, and ZEB1 in malignant progression of cancer. Cancer Metastasis Rev 28: 151-166, 2009.

10. Browne G, Sayan AE and Tulchinsky E: ZEB proteins link cell motility with cell cycle control and cell survival in cancer. Cell Cycle 9: 886-891, 2010.

11. Eggers JC, Martino V, Reinbold R, Schäfer SD, Kiesel L, Starzinski-Powitz A, Schüring AN, Kemper B, Greve B and Götte M: microRNA miR-200b affects proliferation, invasiveness and stemness of endometriotic cells by targeting ZEB1, ZEB2 and KLF4. Reprod Biomed Online 32: 434-445, 2016.

12. Wang ZH, Zhang JL, Duan YL, Zhang QS, Li GF and Zheng DL: MicroRNA-214 participates in the neuroprotective effect of resveratrol via inhibiting $\alpha$-synuclein expression in MPTP-induced Parkinson's disease mouse. Biomed Pharmacother 74: 252-256, 2015.

13. Livak KJ and Schmittgen TD: Analysis of relative gene expression data using real-time quantitative PCR and the 2(-Delta Delta C(T)) method. Methods 25: 402-408, 2001.
14. Maegdefessel L, Spin JM, Raaz U, Eken SM, Toh R, Azuma J, Adam M, Nakagami F, Heymann HM, Chernogubova E, et al: miR-24 limits aortic vascular inflammation and murine abdominal aneurysm development. Nat Commun 5: 5214, 2014.

15. Nothnick WB: MicroRNAs and endometriosis: Distinguishing drivers from passengers in disease pathogenesis. Semin Reprod Med 35: 173-180, 2017.

16. Matsuzaki S and Darcha C: Involvement of the Wnt/ $\beta$-catenin signaling pathway in the cellular and molecular mechanisms of fibrosis in endometriosis. PLoS One 8: e76808, 2013.

17. Tanwar PS, Lee HJ, Zhang L, Zukerberg LR, Taketo MM, Rueda BR and Teixeira JM: Constitutive activation of Beta-catenin in uterine stroma and smooth muscle leads to the development of mesenchymal tumors in mice. Biol Reprod 81: 545-552, 2009

18. Zhang L, Xiong W, Xiong Y, Liu H and Liu Y: 17 beta-Estradiol promotes vascular endothelial growth factor expression via the Wnt $/ \beta$-catenin pathway during the pathogenesis of endometriosis. Mol Hum Reprod 22: 526-535, 2016.

19. Matsuzaki $S$ and Darcha C: In vitro effects of a small-molecule antagonist of the Tcf/ß-catenin complex on endometrial and endometriotic cells of patients with endometriosis. PLoS One 8: e61690, 2013

20. Wang S and Ren D: Allicin protects traumatic spinal cord injury through regulating the HSP70/Akt/iNOS pathway in mice. Mol Med Rep 14: 3086-3092, 2016.

21. Furuya M, Masuda H, Hara K, Uchida H, Sato K, Sato S, Asada H, Maruyama T, Yoshimura Y, Katabuchi H, et al: ZEB1 expression is a potential indicator of invasive endometriosis. Acta Obstet Gynecol Scand 96: 1128-1135, 2017.

22. Panda H, Pelakh L, Chuang TD, Luo X, Bukulmez O and Chegini N: Endometrial miR-200c is altered during transformation into cancerous states and targets the expression of ZEBs, VEGFA, FLT1, IKKbeta, KLF9, and FBLN5. Reprod Sci 19: 786-796, 2012 